\title{
PAPER
}

\section{Evidence of thalamic disinhibition in patients with hemichorea: semiquantitative analysis using SPECT}

\author{
J-S Kim, K-S Lee, K-H Lee, Y-I Kim, B-S Kim, Y-A Chung, S-K Chung
}

J Neurol Neurosurg Psychiatry 2002;72:329-333

See end of article for authors' affiliations

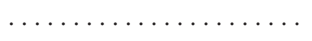

Correspondence to: Dr K-S Lee, Movement Clinic, Department of Neurology, Kangnam St Mary's Hospital, 505 Banpo-Dong, Seocho-Ku, Seoul, 130-701, South Korea;

ks1007@cmc.cuk.ac.kr

Received 10 August 2000 In final revised form 29 October 2001

Accepted

5 November 2001

\begin{abstract}
Objectives: Hemichorea sometimes occurs after lesions that selectively involve the caudate nucleus, putamen, and globus pallidus. Some reports have hypothesised that the loss of subthalamic nucleus control on the internal segment of the globus pallidus, followed by the disinhibition of the thalamus may contribute to chorea. However, the pathophysiology is poorly understood. Therefore, clinicoradiological localisation was evaluated and a comparison of the haemodynamic status of the basal ganglia and thalamus was made.

Methods: Six patients presenting with acute onset of hemichorea were assessed. Neuroimaging studies, including MRI and SPECT examinations in addition to detailed biochemical tests, were performed. A semiquantitative analysis was performed by comparing the ratio of blood flow between patients and normal controls. In addition, the ratio of perfusion asymmetry was calculated as the ratio between each area contralateral to the chorea and that homolateral to the chorea. The comparison was made with a two sample $t$ test.

Results: The causes of hemichorea found consisted of four cases of acute stroke, one non-ketotic hyperglycaemia, and one systemic lupus erythematosus. Brain MRI indicated lesion sites in the contralateral putamen, globus pallidus, caudate nucleus, and subthalamic nucleus. A significant decrease in the ratio of blood flow in the basal ganglia contralateral to the chorea and a significant increase in the thalamus was found when comparing the perfusion asymmetries, which were calculated as the ratio of cerebral blood flow (CBF) for each region to that in the homolateral occipital area $(p<0.05)$.

Conclusion: An alteration in CBF in both the contralateral thalamus and basal ganglia reflect the loss of pallidal inhibitory input from the pallidum to the thalamus. This change in CBF may be one of epiphenomena, which implicates an occurrence of hemichorea in humans.
\end{abstract}

$\mathrm{H}$ emichorea occurs suddenly, and is often accompanied by proximal ballistic movement, which may be due to a wide variety of degenerative, vascular, metabolic, or toxic disorders selectively involving the contralateral caudate nucleus, putamen, and globus pallidus. ${ }^{12}$ Additionally, various structural lesions in the contralateral subthalamic nucleus (STN) and the pallidosubthalamic pathway seem to play a critical part in the development of chorea ${ }^{3-5}$ with an acute vascular accident being the most common pathological process. ${ }^{5}$

A widely accepted hypothesis used to explain these hyperkinetic disorders suggests that the loss of STN control on the internal segment of the globus pallidus (GPi) is followed by the disinhibition of the thalamus. ${ }^{6}$ These explanations are in line with experimental data, including the fact that STN lesions result in dyskinesia, ${ }^{7}$ and that such lesions are associated with reduced levels of spontaneous discharge in the GPi. ${ }^{8}$ However, there are observations that are difficult to reconcile with simple models of basal ganglia function based primarily on the concept of thalamic disinhibition. ${ }^{910}$

Such models are also able to account for hypokinetic disorders on the basis of excessive basal ganglia outflow. In primates with parkinsonian akinesia induced experimentally, the discharge rate of neurons in the GPi as well as in the STN has been found to be abnormally high. ${ }^{11}{ }^{12}$ This is consistent with thalamic disinhibition models showing that akinesia in humans with idiopathic Parkinson's disease can be relieved by selective stereotactic GPi lesions. ${ }^{13}$

The distribution of ${ }^{99 \mathrm{~m}} \mathrm{Tc}$ - hexamethylpropylene aminoxime (HMPAO) within the brain is proportional to cerebral blood flow (CBF). ${ }^{14}$ When cerebral autoregulation is preserved, the $\mathrm{CBF}$ is directly related to metabolic needs, which in turn depends on neuronal functional activity. ${ }^{15}$ However, since the static HMPAO single photon emission computed tomography
(SPECT) did not allow the absolute quantification of the regional $\mathrm{CBF}$, there are some limitations in comparing directly the relative perfusion ratios in the region of interest between normal and pathological conditions. Therefore, we speculated this movement disorder to have an asymmetric pathology on the contralateral hemisphere because hemichorea interchangeably involves one side of the body. Semiquantitative analysis was performed by calculating the ratio of perfusion asymmetry between each area contralateral and homolateral to the chorea.

\section{SUBJECTS AND METHODS}

Six patients (four men, two women) with an age range from 27 to 73 presented with hemichorea. Routine laboratory tests included the measurement of serum glucose, electrolytes, and urinary ketone concentrations. A brain MRI using a 1.5 Tesla superconducting system (Magnetom, Siemens) was obtained 3 days after onset of abnormal movement. The aetiologies of chorea in each patient were classified by laboratory and neuroimaging data. Another six age matched subjects without any other neurological diseases served as normal controls.

A brain SPECT was performed after intravenous injection of $740 \mathrm{MBq}{ }^{99 \mathrm{~m}} \mathrm{Tc}$ HMPAO with lorazepam to reduce movement artifacts. However, the chorea was not completely abolished on examination. The data were obtained with a two head rotating gamma camera (MS II, Siemens, Germany) with a fanbeam collimator. A full $360^{\circ}$ rotation was acquired with a

Abbreviations: CBF, cerebral blood flow; GABA, $\gamma$-aminobutyric acid; GPe, external globus pallidus; GPi, internal globus pallidus 

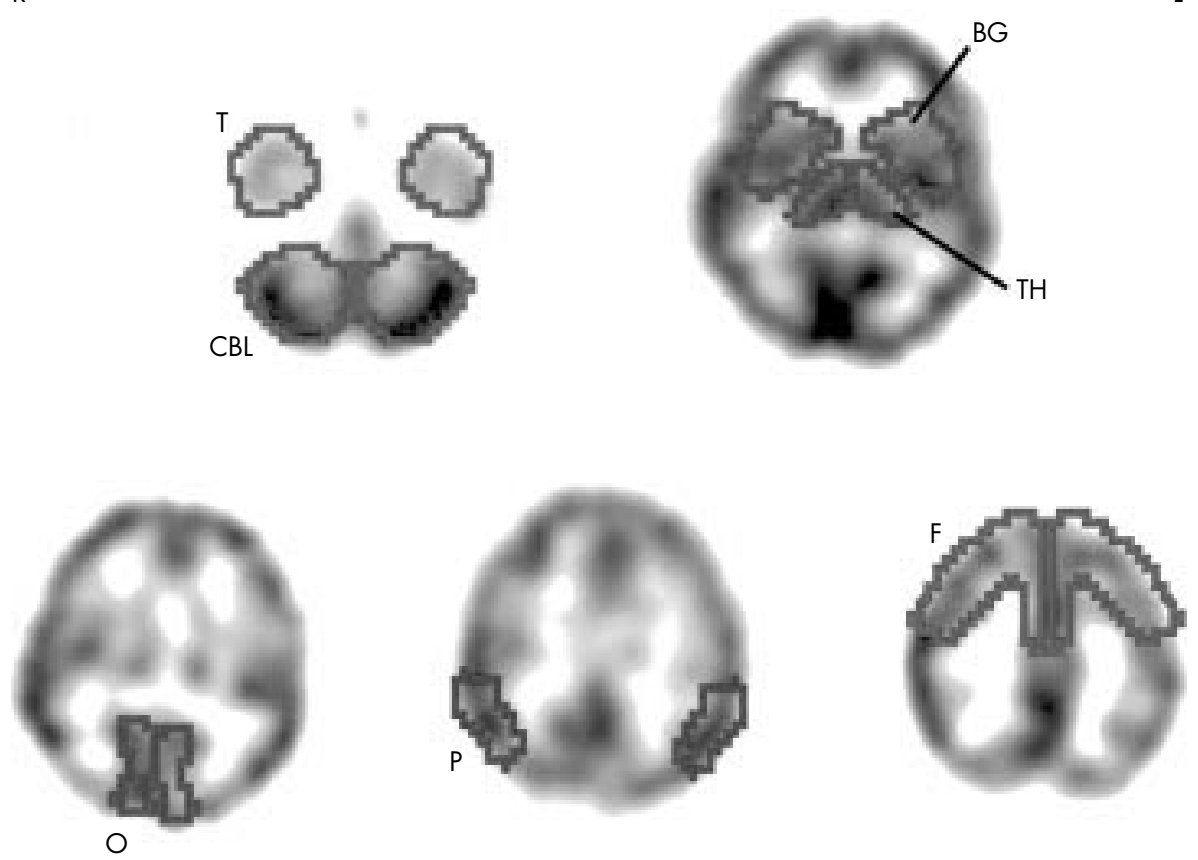

Figure 1 SPECT images showing representative slices of the basal ganglia, thalamus, cerebellum, frontal, temporal, parietal, and the occipital areas in a control subject. The regions of interest were symmetrically drawn on the selected slices based on MRI guidance. CBL, cerebellum; BG, basal ganglia; $T H$, thalamus; $O$, occipital; $P$, parietal; $T$, temporal; $F$, frontal; $R$, right; $L$, left.

matrix of $128 \times 128.10 \mathrm{~mm}$ thick axial slices, tilted along the orbitofrontal line, were reconstructed by a Butterworth filtered backprojection.

Analysis was performed both visually and quantitatively. Two experienced nuclear medicine physicians who were unaware of the patient's clinical state, reviewed all the SPECT results.

\section{Semiquantitative analysis}

${ }^{99 m}$ Tc-HMPAO uptake in the basal ganglia, thalamus, cerebellum, frontal, parietal, temporal and occipital area was quantified by placing appropriate regions of interest (ROI) on positions standardised under MRI guidance (fig 1). Two observers, blind to the clinical state, performed all quantitative analyses.

The analyses were performed by comparing the uptake in the region under evaluation with the uptake in a region unaf- fected by the disease. The occipital area was pathologically spared in the chorea and used as the referenced region. ${ }^{16}$ The $\mathrm{CBF}$ for each region was expressed as the ratio of HMPAO uptake in that region to that in the homolateral occipital area and the CBF ratios were compared between the patients with hemichorea and the normal controls. Additionally, the ratio of perfusion asymmetry was calculated as the ratio between each area contralateral and homolateral to the chorea. A comparison was made with a two sample $t$ test.

\section{RESULTS}

\section{Clinical and neuroimaging data}

Table I summarises the clinical, biochemical, and brain MRI results. The causes of the hemichorea found consisted of four patients with acute stroke (patients 2-5), one with nonketotic hyperglycaemia (patient 6), and one with systemic

\begin{tabular}{|c|c|c|c|c|c|c|}
\hline & \multicolumn{6}{|c|}{ Patient No } \\
\hline & 1 & 2 & 3 & 4 & 5 & 6 \\
\hline Age $(y)$ & 27 & 66 & 53 & 73 & 73 & 67 \\
\hline Side limb & $\mathrm{L}$ & L & $\mathrm{L}$ & L & $\mathrm{R}$ & $\mathrm{L}$ \\
\hline Lesion site & No & $\begin{array}{l}\text { R P, } \\
\text { R GPe }\end{array}$ & $\begin{array}{l}\text { R C, } \\
\text { R P, } \\
\text { R GPe }\end{array}$ & $\begin{array}{l}\text { R GPe, } \\
\text { R STN }\end{array}$ & $\begin{array}{l}\text { LC, } \\
\text { L STN }\end{array}$ & $\begin{array}{l}\text { R GPi, } \\
\text { R STN, } \\
\text { R C }\end{array}$ \\
\hline MRI TI & Iso & Iso & Iso-low & Iso & Iso & High \\
\hline MRI T2 & Iso & High & High & High & High & High \\
\hline Size of lesion $\left(\mathrm{cm}^{3}\right)$ & NC & 4.5 & 5.5 & 4 & 4.5 & 6 \\
\hline Time of SPECT examination (days) & 3 & 4 & 7 & 3 & 4 & 6 \\
\hline Degree of response to treatment & Good & Good & Fair & Good & Good & Good \\
\hline Diabetes $(y)$ & No & No & No & 6 & No & 12 \\
\hline Glucose (mg/dl) & 96 & 112 & 123 & 325 & 99 & 311 \\
\hline $\mathrm{HbAlc}(\%)$ & NR & NR & NR & 10.3 & NR & 7.8 \\
\hline Clinical diagnosis & Vasculitis & Infarct & Infarct & Infarct & Infarct & $\begin{array}{l}\text { Petechial } \\
\text { haemorrhage }\end{array}$ \\
\hline Underlying disease & SLE & HBP & & DM & HBP & DM \\
\hline
\end{tabular}

L, Left; R, right; P, putamen; C, caudate nucleus; GPi, internal pallidum; GPe, external pallidum; STN, subthalamic nucleus; NR, not recorded; NC, not checkable; SLE, systemic lupus erythematosus; HBP, hypertension; DM, diabetes mellitus. 

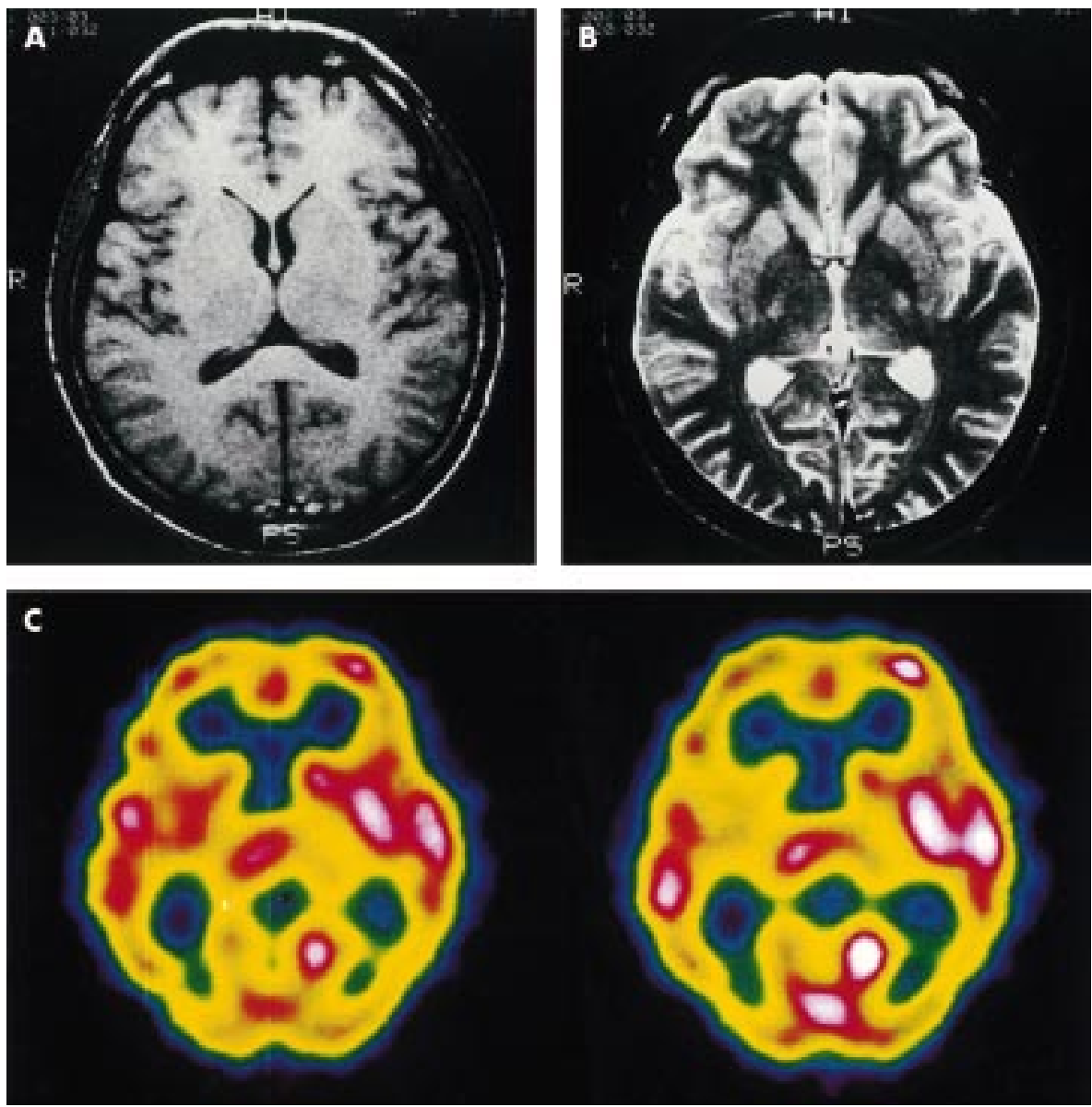

Figure $2 \mathrm{MRI}$ of patient 1 with a left hemichorea ((A) T1 weighted, (B) T2 weighted) showed no definite signal changes in the right basal ganglia or thalamus. However, HMPAO SPECT (C) showed a CBF increase in the right thalamus and a decrease in the right basal ganglia contralateral to the choreic movement. In addition, the left cerebellum showed mildly decreased blood flow suggesting crossed cerebellar diaschisis.

lupus erythematosus (patient 1). Five patients (2-6) had a hemichorea involving the contralateral putamen, caudate nucleus, globus pallidus, and subthalamic nucleus. Patient 1 , with systemic lupus erythematosus, had a hemichorea in the left limbs, but showed no definite anatomical lesion on brain MRI (fig $2 \mathrm{~A}$ and B). All patients received haloperidol (2-10 $\mathrm{mg}$ ) and patient $\mathrm{l}$ was prescribed $60 \mathrm{mg}$ oral prednisolone. The chorea subsided within 1-2 weeks after treatment.

\section{Visual analysis}

The brain SPECT showed a decreased blood perfusion of the basal ganglia and an increased perfusion of the thalamus contralateral to the side of the choreic movement in all patients (fig 2 C). Patients 1, 3, 5, and 6 also had mildly decreased perfusion over the homolateral cerebellar hemisphere and patient 2 had increased perfusion over the right temporal region and patient 5 had decreased perfusion over the left parietal region. In the occipital area, no perfusion asymmetry was found. In addition, there was no perfusion defect in the controls.

\section{Semiquantitative analysis}

Table 2 summarises the blood flow ratios in the basal ganglia and thalamus referenced to that in the homolateral occipital areas. The means (SD) of the ratio in the basal ganglia and thalamus contralateral to the choreic movement were 0.88 $(0.05)$ and $0.95(0.06)$, respectively, and those for the region homolateral to the chorea were $0.97(0.06)$ and $0.90(0.06)$, respectively. By contrast with the patient group, the means (SD) of the right and left basal ganglia and thalamus blood flow in the controls were $0.98(0.08), 0.96(0.07)$ and 0.99 $(0.03), 1.01(0.03)$, respectively. When comparing the percentage of perfusion asymmetry with the normal controls, a significant decrease was found in the ratio of the flow of the basal ganglia contralateral to the chorea $(p=0.014)$ and a significant increase was seen in the thalamus $(p=0.001)$. However, there was no significant difference in the ratio of blood flow over the basal ganglia $(p=0.074)$ and thalamus $(p=0.218)$ contralateral to the chorea compared with that in the control group. Furthermore, no other CBF abnormalities were detected in the frontal, parietal, temporal, and cerebellar areas $(\mathrm{p}>0.05)$.

\section{DISCUSSION}

Experimental hemichorea models can be produced by injecting a $\gamma$-aminobutyric acid (GABA) antagonist into the external segment of the globus pallidus (GPe) or STN. ${ }^{17}{ }^{18}$ The interruption of GABAnergic transmission from the striatum to the GPe would lead to abnormally increased GPe neuron activity, which exerts inhibitory action on the STN. ${ }^{19}$ Increased STN inhibition would result in the loss of its control on the GPi. Besides excitatory STN inputs, ${ }^{20}$ the GPi neurons also receive inhibitory afferent inputs directly from the striatum. The imbalance between the indirect excitatory and direct inhibitory pathways ultimately leads to a disinhibition of the motor thalamus.

However, this simple pathophysiological model of the chorea has rarely been reported in humans. There are only two 
Table 2 SPECT findings for patient group

\begin{tabular}{|c|c|c|c|c|c|c|c|c|}
\hline \multirow[b]{2}{*}{ No } & \multirow{2}{*}{\multicolumn{4}{|c|}{ Visual analysis: }} & \multicolumn{4}{|c|}{ Semiquantitative analysis } \\
\hline & & & & & R TH/ R OC & L TH/ L OC & R TH/ L TH & R BG/ R OC \\
\hline Pl & \multicolumn{4}{|c|}{$\downarrow$ in $R B G, \uparrow$ in $R T H, \downarrow$ in $L C B L, N A$ in $P A, T E, O C, F R$} & 0.92 & 0.83 & 1.09 & 0.92 \\
\hline P2 & \multicolumn{4}{|c|}{$\downarrow$ in $R B G, \uparrow$ in $R T H, \uparrow$ in $R T E, N A$ in CBL, PA, OC, FR } & 0.86 & 0.83 & 1.05 & 0.84 \\
\hline P3 & \multicolumn{4}{|c|}{$\downarrow$ in $R B G, \uparrow$ in $R$ TH, $\downarrow$ in L CBL, NA in PA, TE, OC, FR } & 0.93 & 0.88 & 1.08 & 0.82 \\
\hline P4 & \multicolumn{4}{|c|}{$\downarrow$ in $R B G, \uparrow$ in $R T H, N A$ in $C B L, P A, T E, O C, F R$} & 1.12 & 0.98 & 1.14 & 0.96 \\
\hline P5 & \multicolumn{4}{|c|}{$\downarrow$ in $L B G, \uparrow$ in $L T H, \downarrow$ in $R C B L, \downarrow$ in $L P A, T E, N A$ in $O C, F R$} & 0.93 & 0.98 & 0.95 & 0.94 \\
\hline P6 & \multicolumn{4}{|c|}{$\downarrow$ in $R B G, \uparrow$ in $R T H, \downarrow$ in $L C B L, N A$ in $P A, T E, O C, F R$} & 1.01 & 0.93 & 1.08 & 0.88 \\
\hline $\mathrm{Cl}$ & \multicolumn{4}{|l|}{ NA } & 0.96 & 0.97 & 0.96 & 0.86 \\
\hline $\mathrm{C} 2$ & \multicolumn{4}{|l|}{ NA } & 1.03 & 1.05 & 0.99 & 0.95 \\
\hline $\mathrm{C} 3$ & \multicolumn{4}{|l|}{ NA } & 0.98 & 0.99 & 0.95 & 1.07 \\
\hline $\mathrm{C} 4$ & \multicolumn{4}{|l|}{ NA } & 1.02 & 1.02 & 0.95 & 1.04 \\
\hline C5 & \multicolumn{4}{|l|}{ NA } & 0.98 & 0.99 & 0.96 & 0.96 \\
\hline \multirow[t]{2}{*}{$\mathrm{C} 6$} & \multicolumn{4}{|l|}{ NA } & 0.99 & 1.01 & 1.06 & 0.97 \\
\hline & \multicolumn{8}{|c|}{ Semiquantitative analysis: } \\
\hline No & L BG/L OC & R BG/L BG & $\mathrm{R} C B L / R O C$ & $\mathrm{~L} \mathrm{CBL/L} \mathrm{OC}$ & R CBL/L CBL & R FR/R OC & L FR/L OC & R FR/ L FR \\
\hline P1 & 0.95 & 0.95 & 0.89 & 0.86 & 1.01 & 0.81 & 0.82 & 0.97 \\
\hline P2 & 0.90 & 0.94 & 0.86 & 0.85 & 1.02 & 0.79 & 0.80 & 1.00 \\
\hline P3 & 0.98 & 0.85 & 0.89 & 0.85 & 1.07 & 0.82 & 0.82 & 1.02 \\
\hline P4 & 1.01 & 0.95 & 0.93 & 0.92 & 1.01 & 0.84 & 0.82 & 1.02 \\
\hline P5 & 0.88 & 1.07 & 0.94 & 0.99 & 0.95 & 0.85 & 0.91 & 0.94 \\
\hline P6 & 0.97 & 0.90 & 0.98 & 0.95 & 1.02 & 0.84 & 0.85 & 0.98 \\
\hline $\mathrm{Cl}$ & 0.86 & 0.97 & 1.14 & 1.11 & 1.00 & 0.93 & 0.90 & 1.00 \\
\hline $\mathrm{C} 2$ & 0.94 & 1.02 & 0.99 & 0.99 & 1.01 & 0.92 & 0.90 & 1.03 \\
\hline C3 & 1.04 & 0.99 & 0.92 & 0.88 & 1.01 & 0.87 & 0.83 & 1.01 \\
\hline $\mathrm{C} 4$ & 1.02 & 0.97 & 0.97 & 0.93 & 0.99 & 0.88 & 0.83 & 1.01 \\
\hline C5 & 0.92 & 1.01 & 0.90 & 0.85 & 1.03 & 0.85 & 0.84 & 0.98 \\
\hline \multirow[t]{3}{*}{ C6 } & 0.98 & 1.07 & 0.95 & 1.05 & 0.98 & 0.85 & 0.92 & 1.00 \\
\hline & \multicolumn{8}{|c|}{ CBF analysis: } \\
\hline & $\mathrm{R} P A / \mathrm{R} O C$ & L PA/L OC & R PA/L PA & R TE/R OC & $\mathrm{L} T E / \mathrm{L} \mathrm{OC}$ & R TE/L TE & $\mathrm{ROC} / \mathrm{LOC}$ & \\
\hline $\mathrm{Pl}$ & 0.90 & 0.91 & 0.97 & 0.82 & 0.79 & 1.02 & 0.98 & \\
\hline P2 & 1.02 & 0.99 & 1.04 & 0.85 & 0.75 & 1.14 & 1.01 & \\
\hline P3 & 1.11 & 1.06 & 1.07 & 0.81 & 0.90 & 0.92 & 1.02 & \\
\hline P4 & 1.09 & 1.13 & 0.96 & 0.90 & 0.96 & 0.94 & 1.00 & \\
\hline P5 & 0.94 & 0.83 & 1.13 & 0.85 & 0.76 & 1.12 & 1.00 & \\
\hline P6 & 1.08 & 1.06 & 1.01 & 0.95 & 0.94 & 1.00 & 0.99 & \\
\hline $\mathrm{Cl}$ & 0.93 & 0.98 & 0.92 & 0.81 & 0.74 & 1.06 & 0.97 & \\
\hline $\mathrm{C} 2$ & 1.14 & 1.13 & 1.02 & 0.94 & 0.91 & 1.04 & 1.01 & \\
\hline C3 & 1.13 & 1.08 & 1.01 & 0.87 & 0.79 & 1.06 & 0.96 & \\
\hline $\mathrm{C} 4$ & 1.05 & 0.97 & 1.03 & 0.83 & 0.83 & 0.95 & 0.95 & \\
\hline C5 & 0.88 & 0.86 & 0.99 & 0.75 & 0.75 & 0.97 & 0.97 & \\
\hline C6 & 0.95 & 1.01 & 1.02 & 0.77 & 0.85 & 0.98 & 1.08 & \\
\hline
\end{tabular}

$L$, left; $R$, right; $B G$, basal ganglia; $T H$, thalamus; $C B L$, cerebellum; $O C$, occipital area; $F R$, frontal area; $P A$, parietal area; $T E$, temporal area; $\downarrow, \uparrow$, decrease or increase in blood flow compared with contralateral side; NA, no asymmetry.

The ratio is expressed as the investigated regional blood flow for each region referenced to that the homolateral occipital area.

reports showing an increase in thalamic perfusion after a striatal ischaemic lesion $^{21}$ or an increase in thalamic glucose metabolism in Huntington's disease. ${ }^{22}$ Moreover, there are observations that are difficult to reconcile with these simple models of basal ganglia function based primarily on the concept of thalamic disinhibition. One of the most noteworthy discrepancies involves the fact that lesions of the basal ganglia output nuclei do not result in dyskinesia.' Another related problem in thalamic disinhibition theories is that lesions of the ventrolateral thalamus do not result in akinesia. Yet, according to such theories parkinsonian akinesia is generally attributed to increased basal ganglia outflow, which results in excessive inhibition at the ventrolateral thalamus level. ${ }^{6}$ One possible explanation for this apparent discrepancy is that the functional relevance of the descending basal ganglia outflow to the pedunculopontine nucleus may have been underestimated. In this case, lesions of the thalamus that only block the re-entrant influence of the basal ganglia, may leave intact those descending influences that are conveyed directly to the segmental motor apparatus.
Both SPECT and HMPAO were employed to obtain a functional status of the patients with hemichorea. Although a comparison of the ratio of blood flow over the basal ganglia and thalamus contralateral to the chorea with that in the control group was non-significant, our finding of perfusion asymmetry in the thalamus and the basal ganglia contralateral to the hemichorea was of particular interest because it may reflect the loss of pallidal inhibitory input to the thalamus. The $\mathrm{CBF}$ increase seen in the thalamus contralateral to the choreic movement probably reflects increased thalamic neuronal activity, free from inhibitory pallidal control. In addition, as seen in patient 1, a brain SPECT has many advantages over a brain MRI in detecting functional abnormalities in chorea.

Because basal ganglia influence movement primarily through their projections to the primary or secondary areas of the motor cortex, some differences in cortical perfusion were examined in patients with hemichorea. However, in the cortical areas, our patients did not show any differences in the ratio of perfusion contralateral or homolateral to the hemichorea. There have been several studies of the cortical physiological 
mechanism concerning hyperkinetic disorders. However, the function of the thalamocortical projections and cortical areas, leading to chorea, remains unclear and some controversy still exists. ${ }^{23-26}$ Although our findings did not disclose the metabolic rate but the relative perfusion rate in the cortical area, this suggests that the chorea is produced by activation of an intact motor cortex by an abnormal or immature trigger pulse in the basal ganglia, which is in agreement with the original proposal by Kanazawa. ${ }^{27}$ Studying cortical metabolism in patients with hemichorea using positron emission tomography may be necessary.

We postulate that the primary event is a vascular insult in the striatum, leading to a dysfunction of the GABAnergic projection neurons. Consequently, damage to the striatum causes a contralateral hemichorea when the striatal neurons that project through the GPe to the STN are selectively affected. In summary, our interpretation of the hyperperfusion of the thalamus found is in accordance with one of an epiphenomenon, which implicates the occurrence of hemichorea in humans.

\section{ACKNOWLEDGEMENT}

This work was supported by grants from the Catholic Medical Center Research Foundation.

\section{Authors' affiliations}

J-S Kim, K-S Lee, K-H Lee, Y-I Kim, B-S Kim, Department of Neurology, College of Medicine, The Catholic University of Korea Y-A Chung, S-K Chung, Department of Nuclear Medicine

\section{REFERENCES}

1 Kase CS, Maulsby GO, deJuan E, et al. Hemichorea-hemiballism and lacunar infarction in the basal ganglia. Neurology 1981;31:452-5.

2 Shan DE, Ho DMT, Chang C, et al. Hemichorea-hemiballism: an explanation for MR signal changes. Am J Neuroradiol 1998;19:863-70.

3 Martin TP, Akock NS. Hemichorea associated with a lesion of corpus luysii. Brain 1934;57:504-16.

4 Hyland $\mathbf{H}$, Foroman DM. Prognosis in hemiballism. Neurology 1957;7:381-91.

5 Dewey RB, Jankovic J. Hemiballism-hemichorea: clinical and pharmacological findings in 21 patients. Arch Neurol 1989:46:862-7.

6 DeLong MR. Primates models of movement disorders of basal ganglia origin. Trends Neurosci 1990;40:281-5.

7 Carpenter MB, Whittier JR, Mettler FA. Analysis of choreoid hyperkinesia in the rhesus monkey: surgical and pharmacological analysis of hyperkinesia resulting from lesions in the subthalamic nucleus of Luys. J Comp Neurol 1950:92:293-322.

8 Bergman H, Wichmann T, Delong MR. Reversal of experimental parkinsonism by lesions of the subthalamic nucleus. Science 1990;249:1436-8.
9 Mink JW, Thach WT. Basal ganglia motor control. III. Pallidal ablation: normal reaction time, muscle cocontraction, and slow movement. $J$ Neurophysiol 1991;65:330-51.

10 Steriade $\mathbf{M}$, Llinas RR. The functional states of the thalamus and the associated neuronal interplay. Physiol Rev 1988;68:649-742.

11 Miller WC, Delong MR. Parkinsonian symptomatology: an anatomical and physiological analysis. Ann NY Acad Sci 1988;515:287-302.

12 Filion M, Tremblay L, Bedard PJ. Abnormal influences of passive limb movement on the activity of globus pallidus neurons in parkinsonian monkeys. Brain Res 1988;444:165-76.

13 Laitinen LV, Bergenheim AT, Hariz MI. Leksell's posteroventral pallidotomy in the treatment of Parkinson's disease. J Neurosurg 1992;76:53-61.

14 Sharp PF, Smith FW, Gemmell HG, et al. Technetium-99m HM-PAO stereoisomers as potential agents for imaging regional cerebral blood flow: human volunteer studies. J Nucl Med 1986;27:171-7.

15 Raichle ME, Grubb RL, Gado MH, et al. Correlations between regional cerebral blood flow and oxidative metabolism. Arch Neurol 1976;33:523-6.

16 Chang MH, Li JY, Lee SR, et al. Non-ketotic hyperglycaemic chorea: a SPECT study. J Neurol Neurosurg Psychiatry 1996;60:428-30.

17 Crossman AR, Mitchell IJ, Sambrook MA, et al. Chorea and myoclonus in the monkey induced by gamma-aminobutyric acid antagonism in the lentiform complex. Brain 1988;111:1211-33.

18 Crossman AR, Sambrook MA, Jackson A. Experimental hemichorea/ hemiballism in the monkey: studies on the intracerebral site of action in a drug-induced dyskinesia. Brain 1984; 107:579-96.

19 Ohye C, Le Guyader C, Feger J. Response of subthalamic and pallidal neurons to striatal stimulation: an extracellular study on awake monkeys. Brain Res 1976;111:241-52.

20 Hamada I, DeLong MR. Excitotoxic acid lesions of the primate subthalamic nucleus result in reduced pallidal neuronal activity during active holding. J Neurophysiol 1992;68:1859-66.

21 Pantano P, Di Cesare S, Ricci M, et al. Hemichorea after a striatal ischemic lesion: evidence of thalamic disinhibition using single-photon emission computed tomography: a case report. Mov Disord 1996;11:444-7.

22 Young AB, Penney JB, Starosta-Rubinstein S, et al. PET scan investigations of Huntington's disease: cerebral metabolic correlates of neurological features and functional decline. Ann Neurol 1986;20:296-303.

23 Weeks RA, Ceballos-Braumann A, Piccini P, et al. Cortical control of movement in Huntington's disease: a PET activation study. Brain 1997; 120:1569-78.

24 Kuwert T, Lange HW, Langen KJ, et al. Cortical and subcortical glucose consumption measured by PET in patients with Huntington's disease. Brain 1990; 113:1405-23.

25 Hosokawa S, Ichiya Y, Kuwabara Y, et al. Positron emission tomography in case of chorea with different underlying diseases. J Neurol Neurosurg Psychiatry 1987;50:1284-7.

26 Kuhl DE, Phelps ME, Markham CH, et al. Cerebral metabolism and atrophy in Huntington's disease determined by 18 FDG and computed tomographic scan. Ann Neurol 1982;12:425-34.

27 Kanazawa I. Clinical pathophysiology of basal ganglia disease. In: Vinken PJ, Bruyn GW, Klawans HL, eds. Extrapyramidal disorders. Handbook of clinical neurology. Amsterdam: Elsevier, 1986:65-85. 